\title{
Dopamine-Somatostatin Chimeric Molecule BIM-23A760
}

National Cancer Institute

\section{Source}

National Cancer Institute. Dopamine-Somatostatin Chimeric Molecule BIM-23A760. NCI

Thesaurus. Code C88345.

A chimeric molecule directed against dopamine and somatostatin receptors with potential antineoplastic activity. Combining two pharmacological moieties, a somatostatin analogue and a dopamine agonist, dopamine-somatostatin chimeric molecule BIM-23A760 binds with high affinity to dopamine D2 receptor (D2R) and somatostatin receptor subtype 2 (SST R2), and to a lesser extent to somatostatin receptor subtype 5 (SST R5). This agent appears to exert its effect mainly by binding to D2R to activate the ERK1/2 and P38 MAPK pathways, thus inducing apoptosis and inhibiting cellular proliferation in non-functioning pituitary adenoma (NFPA) and neuroendocrine tumors. By binding to SSTR2, this agent may inhibit the secretion of growth hormone $(\mathrm{GH})$ by the pituitary gland. 